\title{
Commentary
}

Seth M. Levine*

\section{A comment on Morey et al. (2020)}

https://doi.org/10.1515/tnsci-2020-0121

received April 23, 2020; accepted June 1, 2020

Recent functional MRI studies have demonstrated that aversive learning can alter high-dimensional representations of conceptual information in healthy individuals $[1,2]$. Morey and colleagues [3] built on these findings by applying a previously employed fear-conditioning paradigm, in which $50 \%$ of the exemplars of a semantic category (either animals or tools) were paired with an electric shock [1], to a sample of posttraumatic stress disorder (PTSD) patients. Such an approach that utilizes multivariate (i.e., pattern-based) analyses provides the field an additional route for developing functional neuroimaging biomarkers of anxiety disorders.

The univariate (i.e., amplitude-based) results in the article partially echo the authors' expectations (in that PTSD patients yielded a higher signal change for the conditioned category, compared to the neutral category, with respect to trauma-exposed veteran controls) in brain regions previously linked to fear learning [4]. However, the authors additionally expected similar univariate effects and increased pattern similarity in category-selective regions (in the occipitotemporal cortex) for the PTSD patients (ostensibly linked to hyper-reactivity of the salience network), which, interestingly, they did not find. Instead, the dissimilarity matrices displayed in the authors' fourth supplementary figure suggest that any within-category similarity increases in the PTSD group may be negligible.

The effect sizes of these multivariate results appear dramatically reduced (for both the PTSD patients and controls) compared to previously reported within-category similarity increases for healthy individuals in the occipitotemporal cortex [1]. Coupling this deviation with the authors' prediction of stronger pattern-based effects in the patients raises the question: is it not the

\footnotetext{
* Corresponding author: Seth M. Levine, Department of Cognitive and Clinical Neuroscience, Central Institute of Mental Health, Medical Faculty Mannheim, Heidelberg University, 68159 Mannheim, Germany; Department of Psychiatry and Psychotherapy, University of Regensburg, 93053 Regensburg, Germany, e-mail: seth.levine@zi-mannheim.de
}

unexpected nature of the results that renders them even more interesting? Such results suggest a potential distinction in how fear-generalization mechanisms may interact with information processing in fronto-limbic as opposed to occipitotemporal regions when measured with amplitude- versus pattern-based methods; thus, placing null findings into a larger context can foster new theories.

Moreover, that the authors' multivariate results do not show strong generalization within category-selective cortex raises the possibility that the cohort investigated may meaningfully differ from individuals with, for example, no combat exposure or even lower scores on trauma/depression-related questionnaires. As the authors discuss, one might find the "expected" effects in category-selective brain regions when using more ecologically valid (or pertinent) stimuli for PTSD patients. However, turning the argument around leads to the idea that, despite the behavioral results, generalization mechanisms linked to category-selective cortex may fail to engage in trauma-exposed individuals (at least with non-pertinent stimuli); the specifics of such a phenomenon could spark a promising new line of research.

While the authors understandably focus their discussion on the positive results, one should not gloss over the unexpected multivariate results, as they may indicate cognitive differences underlying individuals with PTSD, subclinical PTSD, or a general susceptibility to anxiety disorders (potentially contributing to further subtyping [5]). Ultimately, revolutionizing psychiatry research with functional neuroimaging will require breaking away from traditional expectations and cleverly translating cognitive neuroscience methods (like those employed by Morey and colleagues) to the clinical domain.

Acknowledgments: The author would like to thank Aino L. I. Alahäivälä and Philipp Seidel for comments on an earlier version of this manuscript.

Conflict of interest: The author states no conflict of interest. 


\section{References}

[1] Dunsmoor JE, Kragel PA, Martin A, La Bar KS. Aversive learning modulates cortical representations of object categories. Cerebral Cortex. 2014;24(11):2859-72. doi: 10.1093/cercor/bht138.

[2] Levine SM, Pfaller M, Reichenberger J, Shiban Y, Mühlberger A, Rupprecht R, Schwarzbach JV. Relating experimentally-induced fear to pre-existing phobic fear in the human brain. Social Cogn Affect Neurosci. 2018;13(2):164-172. doi:10.1093/scan/nsx147.

[3] Morey RA, Haswell CC, Stjepanović D, Mid-Atlantic MIRECC Workgroup, Dunsmoor JE, LaBar KS. Neural correlates of conceptuallevel fear generalization in posttraumatic stress disorder.
Neuropsychopharmacology. 2020. doi: 10.1038/s41386-0200661-8.

[4] Morey RA, Dunsmoor JE, Haswell CC, Brown VM, Vora A, Weiner J, et al. Fear learning circuitry is biased toward generalization of fear associations in posttraumatic stress disorder. Transl Psychiatry. 2015;5(12):e700. doi: 10.1038/tp.2015.196.

[5] Nicholson AA, Densmore M, McKinnon MC, Neufeld RW], Frewen PA, Théberge J, et al. Machine learning multivariate pattern analysis predicts classification of posttraumatic stress disorder and its dissociative subtype: a multimodal neuroimaging approach. Psychol Med. 2019;49(12):2049-59. doi: $10.1017 /$ S0033291718002866. 